Chapter 14

\title{
Cells and Holograms - Holograms and Digital Holographic Microscopy as a Tool to Study the Morphology of Living Cells
}

\author{
Kersti Alm, Zahra El-Schich, Maria Falck Miniotis, \\ Anette Gjörloff Wingren, Birgit Janicke and \\ Stina Oredsson
}

Additional information is available at the end of the chapter

http://dx.doi.org/10.5772/54505

\section{Introduction}

Digital holographic microscopy (DHM) is an emerging high-resolution imaging technique that offers real-time imaging and quantitative measurements of physiological parameters without any staining or labeling of cells. The first DHM images of living cells were obtained 8-10 years ago [1, 2]. Analysis of human hepatocytes showed that DHM was a versatile tool for in vivo cell analysis by using quantitative amplitude and phase-contrast imaging with very high resolution [1]. Another study showed that the quantitative distribution of the optical path length created by transparent specimens contained information concerning both morphology and refractive index of the observed mouse cortical neurons [2]. This could only be measured by DHM and not by phase contrast and Nomarski's differential interference contrast (DIC) microscopy. In addition, the high sensitivity of these phase-shift measurements enables sub-wavelength axial accuracy, offering attractive possibilities for the visualization of cellular dynamics.

Since the first studies on living cells were performed, DHM has been used to study a wide range of different cell types, e.g. protozoa, bacteria and plant cells, mammalian cells such as nerve cells, stem cells, various tumor cells, bacterial-cell interactions, red blood cells and sperm cells [3-18].

The phase shift caused by cells is dependent on the amount of nonaqueus material, which affects the refractive index [19]. Thus, changes in cellular water concentration will dilute or 
concentrate the nonaqueus material resulting in phase shift changes. Jourdain et al., exploited this in a study of water fluxes in neurons elicited by stimulation of glutamate receptors in cultures of cortical neurons isolated from mouse embryos [17]. Glutamate receptor stimulation results in neuronal depolarization with a rapid $\mathrm{Na}^{+}, \mathrm{Ca}^{+}$and $\mathrm{Cl}^{-}$and concomitantly water inflow. Using DHM they found three types of glutamate-induced phase shifts in individual neurons. They also showed that one of these phase shifts resulted in cell death. The role of the two cotransporters NKCC1 and KCC2 was also investigated. These phase shifts were only found in older cultures of neurons but not in young neuronal cultures, which was correlated to the expression of glutamate receptors. Kemper et al., presented a set-up for DHM and algorithms for digital holographic reconstruction, which they used to investigate the shape of three human pancreatic duct adenocarcinoma cell lines [3]. By comparing scanning electron microscopy with DHM, they concluded that DHM gives fast and reliable results on live cell morphology. They also used DHM to investigate cellular dynamic changes taking place during 3 minutes after addition of an actin cytoskeleton-disrupting toxin, showing distinct changes in the cellular refractive index correlating with cell collapse.

There are several diseases where the number of immature red blood cells (reticulocytes) increases. The determination of reticulocyte numbers requires staining with different markers, thus, noninvasive methods would yield faster results. Mihailescu et al., recently developed a code for automated simultaneous individual label free cell image separation using DHM to distinguish between reticulocytes and mature erythrocytes [13]. Red blood cells are imaged as donut-like structures using DHM [5] and the obtained data can be used to calculate erythrocyte volume. Hemoglobin is the main nonaqueus material in erythrocytes which causes a phase shift. The resulting phase signal can be directly related to the mean corpuscular hemoglobin [20]. Rappaz et al., showed that DHM could in fact be used to calculate the mean corpuscular hemoglobin concentration of erythrocytes [5]. Similar results have also been presented by Jin et al. [14].

Sperm morphology has been identified as a characteristic that can be useful in the prediction of fertilizing capacity. Recently it has been shown that DHM can be used to monitor the structure and composition of sperm heads [11-12] and that there can be significant differences between individuals [12]. The data are interesting and should stimulate further research into the use of DHM in assessing fertility.

A new tool for the study of bacterial-cell interaction has been described very recently, such as adherence and invasion, which is an interesting application in order to advance diagnostic tools for evaluating infection scenarios [15]. Holographic optical tweezers were used to select, move and align multiple rod shaped Bacillus subtilis DB 630 on top of human pancreatic ductal adenocarcinoma cells and the interaction were subsequently monitored with offaxis DHM. The authors were able to reproduce the alignment of bacteria on the cell surface with very high accuracy, while bacterial morphology and viability remained unaffected.

Excitingly, a portable holographic pixel super-resolution lens-free on-chip microscope has been developed to be used in remote locations, for the purpose of monitoring and diagnosing pathogens in samples such as blood and water [21]. The $95 \mathrm{~g}$ microscope features less than $1 \mu \mathrm{m}$ resolution over a wide field-of view of $\sim 24 \mathrm{~mm}^{2}$. A digital sensor-array 
acquires holograms and pixel super-resolution algorithm post-processing yields high resolution images with retained wide field-of view. A digital micro-processor that enables step-wise LED illumination for lens-free transmission holograms was employed for this purpose. Reconstructed holograms of the human malaria parasite Plasmodium falciparum in smears showed that infected red blood cells can be identified using a combination of amplitude and phase images.

A key feature of DHM is the ability to study cell morphological changes associated with differentiation. In a recent study, Chalut and colleagues set out to investigate the physical changes that are associated with cellular differentiation [22]. They used a rapidly differentiating sub-line of human promyelocytic leukemic cell line HL-60, HL-60/S4, which were induced to differentiate into various mature blood cells; neutrophils, monocytes and macrophages. As shown by off-axis DHM and confocal microscopy, neutrophil and monocyte differentiation displayed a decrease in overall refractory index and a change in the distribution of cellular material towards the nucleus, hence these cells were becoming less dense during differentiation. On the contrary, macrophage differentiation showed no change in the distribution pattern of cellular material, although overall refractory index increased. Moreover, the use of a laser trap technique, optical stretching, showed that neutrophils and monocytes became more compliant (soft), while macrophages became stiffer after just one day of differentiation. The study shows that DHM together with optical stretching can be used as novel approach to monitor and distinguish cellular differentiation of myeloid precursor cells.

The common denominator of all these described studies is that DHM is used to analyze the morphology of living cells in a way that is automatic, cost efficient and causes the cells no harm. Moreover, the method will contribute with large amounts of cell data in a high throughput manner.

\section{Cell death and DHM}

The balance between cell growth and controlled cell death is very crucial for many physiological processes such as embryogenesis, differentiation, in the immune system and in diseases such as stroke, heart failure and neurodegeneration [23]. Cells can die in two ways in vertebrates, either by cell disintegration, necrosis, or by a process of programmed cell death, apoptosis, that play a central role in development and homeostasis. On the contrary, necrosis is a fast induction of cell death that induces an inflammatory response. Late necrosis is characterized by extensive DNA hydrolysis, organelle breakdown, and finally cell lysis. Morphologically, dying cells differ from viable cells in many ways. Apoptosis begins with a variety of morphological alterations: cell membrane changes such as loss of membrane asymmetry and attachment, cell shrinkage, formation of small blebs, nuclear fragmentation, chromatin condensation, chromosomal DNA fragmentation and finally the cell breaks into several apoptotic bodies [24]. One of the earliest indications of apoptosis is the translocation of the membrane phospholipid phosphatidylserine (PS) to the outside of the plasma membrane [25]. 
The most commonly used assays to differentiate between viable and non-viable cells today are trypan blue and propidium iodide, both laborious and time-consuming methods. Khmaladze and colleagues used DHM to measure, with high time and volume resolution and in real-time, cell volume changes induced by apoptosis [26]. Adherent human epithelial KB cells were treated with $1-2 \mu \mathrm{M}$ staurosporine for 4 hours (h). By measuring the phase change of light passing through cells, an early stage morphological feature of apoptosis were observed in treated cells - a marked decrease in cell volume. These observations were consistent with previous studies using standard population-based techniques. The ability to analyze individual cells in a given cell population by using DHM was successfully demonstrated, as individual treatment-induced cell responses could be monitored. Moreover, with the high time resolution that can be obtained using DHM, one can also study initial cell responses. Accordingly, the authors did indeed note time-dependent fluctuations in cell volume, which increased in the earlier phases of treatment.

Excessive stimulation of neurotransmitters through addition of L-glutamate was used to induce cell death in primary cultures of mouse cortical neurons [16]. Cell volume regulation was monitored by DHM phase response, which allowed the researchers to estimate in a very short time-frame, if a neuronal cell would survive or die. By varying the concentration and exposure time of glutamate, the authors could identify reversible phase responses corresponding to phase recovery though an efficient ionic homeostasis. Moreover, they could also observe irreversible phase responses, indicating a constant change of intracellular ionic homeostasis, related to cell volume regulation alterations and suggested as a marker of glutamate induced cell death in neurons. By monitoring the phase shift, the authors were also able to distinguish nuclear condensation and "blebbing" induced by treatment which could indicate that cells were apoptotic rather than necrotic. Importantly, cells recognized within minutes by their DHM phase signal as unable to regulate their ionic homeostasis, were only several hours later identified as dead by trypan blue staining.

\section{The technique}

As opposed to traditional microscopy imaging, DHM is a technique that does not directly result in cell images. Instead, the technique measures the effects on light exerted by a cell. Light that has passed through the cell, and which is affected by the cell, is combined with identical light that has bypassed the cell (Figure 1). This results in the formation of an interference pattern, which is captured by a sensor. From this interference pattern a computer can reconstruct the object (e.g. a cell) that caused the pattern. The creation and capture of an interference pattern requires very low light intensity, thus making the system free from photo toxicity [27].

As the objects are reconstructed in the computer, focusing does not need to be as exact as when capturing cell images directly. The interference pattern, which is the base of the image reconstruction, enables the computer to collect object data from a series of focal planes comprising a calculation span. If the object is within the calculation span, the computer will be able to reconstruct a focused object. 


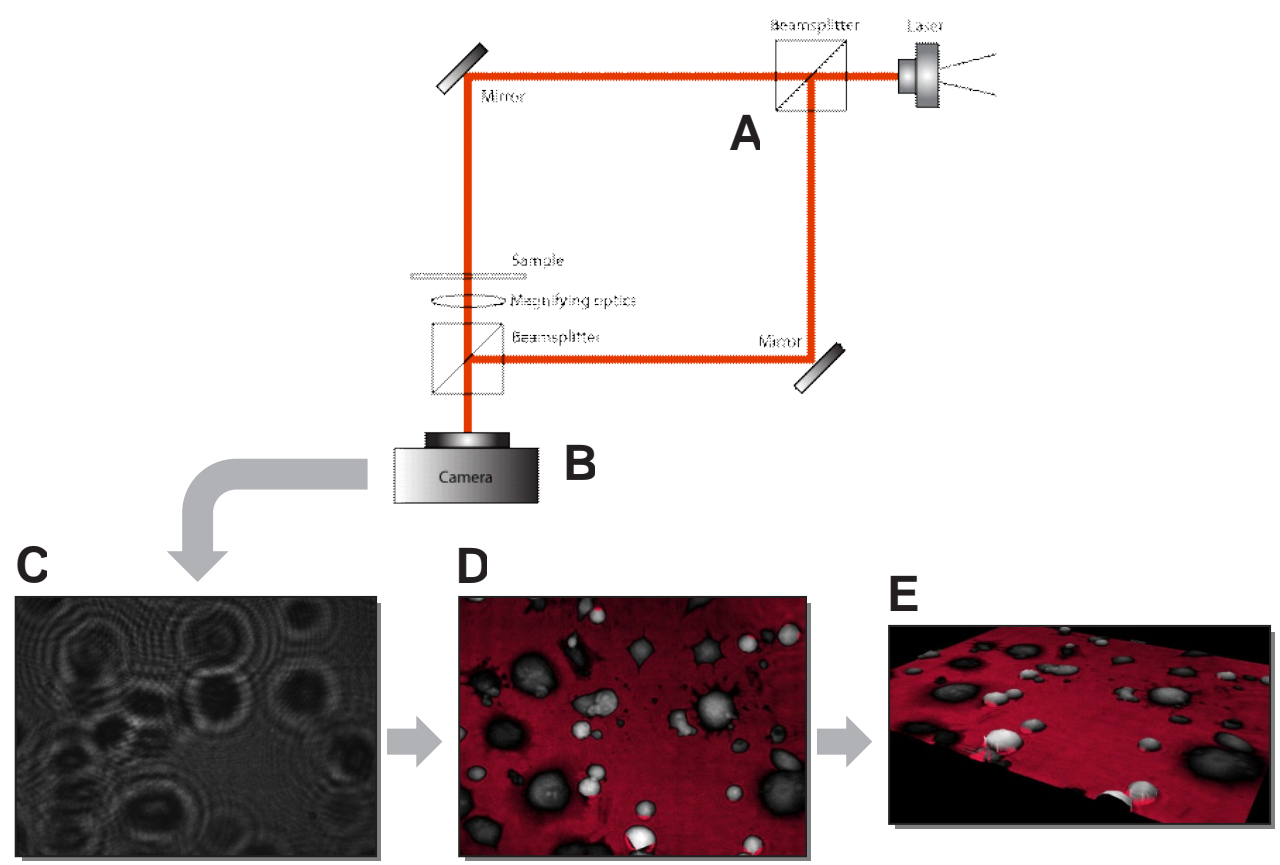

Figure 1. A digital holographic setup. (A) A laser beam is split into two identical beams. One beam passes through an object (in this case the cells) while the other beam travels undisturbed. (B) The two beams are merged again and focused on a camera sensor. (C) The sensor will capture an interference image which is. (D) A computer algorithm reconstructs the objects, which in this figure are $L 929$ mouse fibroblast cells. (E) As the reconstruction will give 3Dinformation, 3D-images can be displayed.

The transmission of light can be compared to the transmission of waves over water. The waves come with different heights and with different intervals. The intensity of light is determined by the height of the light-wave, called the amplitude of the wave. The color of light is determined by the distances between the wave tops, called the wave-length. The human eye can detect the intensity of the light and its color. An additional characteristic of light is of importance, namely in which phase of the light wave the light is detected. In light from a laser source, all light-waves are produced synchronously and simultaneously, i.e. they are coherent and all waves are in the same phase. Regular sunlight on the other hand is noncoherent as the light-waves are not synchronized but come with various patterns of deviation resulting in differences in the phases of the light waves. The human eye cannot detect whether light is coherent or non-coherent. 
If light passes through an object that can absorb light, the amplitude will decrease and thus the intensity decreases. If light passes through an optically dense object, the light will be delayed, and the phase of the light will shift. Using digital holography it is possible to measure the shifts in the phases of light.

As the reconstructed holographic cell images are based on the phase shifts of the light, it is easier to quantify cell characteristics. The area is obtained from the total number of pixels covering the cell being imaged. By determining the edge, the shape of the cell can be disclosed. The shift in the phase of light that occurs when the light passes though the cell can be translated into cellular thickness. The phase shift is related to optical path length and the wavelength of the light. As a consequence, the optical path length is directly correlated to the thickness and the refractory index of the cell. In short, if the phase shift is measured and the wavelength of the light as well as the refractive index of the cell is known, the thickness of the cell can be calculated. Lastly, the volume of the cell can be calculated from the area and the thickness. In other words, DHM enables its user to quantify cellular characteristics without causing photo toxicity. Moreover, as mechanical focusing does not need to be exact, DHM makes time-lapse studies more convenient.

\section{Quantitative imaging of cell morphology}

\subsection{Cell division}

Cells reproduce by division in a process called mitosis. All cells go through a series of welldocumented stages, which are the same for all cells except for egg and sperm cells. The duration of each stage however, differs between cell types and is also due to the environmental conditions and external and internal growth signals.

We have studied the morphology of an individual cell division over time using DHM (HoloMonitor M3, Phase Holographic Imaging AB, PHIAB, Lund, Sweden). The pancreatic cancerous cells, PanC-1 (ATCC CRL-1469), were grown on a IBIDI-micro slide (IBIDI, Martinsried, Germany) in their normal growth medium during the entire study and the micro slide was placed on a heating plate (IBIDI) to retain $37^{\circ} \mathrm{C}$. A time-lapse movie was created with one capture taken every 5 minutes for $72 \mathrm{~h}$ in total, and thereafter one cell was selected for a morphological study, which spanned over $24 \mathrm{~h}$.

In Figure 2, the selected cell is presented as an artificially colored image. The 12 individual sequence images represent a time span of $24 \mathrm{~h}$, starting at $5 \mathrm{~h}$ and ending at $29 \mathrm{~h}$ within the total time lapse. After the division of the mother cell, the two daughter cells were followed in the study.

The first three images show how the mother cell rounds up and organizes its chromatin. The "white bar" on top of the cell at time point $12 \mathrm{~h} 45$ min corresponds to the chromatin just before the separation of the two sets of genes. Twenty minutes later (at time point $13 \mathrm{~h} 05$ $\mathrm{min}$ ) there are two distinct sets of chromosomes moving away from each other. Seen at the same time point is a small elongation of the cell as it continues its path of mitosis. The next 
three images spanning over two hours show how the cell continues the separation of its two sets of chromosomes leading to further elongation, until the cell has become two cells. The daughter cells are still connected to each other via their outer membranes, but are clearly two individual cells. Finally the cells adopt the appearance of mature cells. The spreading out of the daughter cells is not synchronized as the daughter cell to the left in the image spreads out at $17 \mathrm{~h} 25 \mathrm{~min}$ and the right daughter cell at $29 \mathrm{~h}$.

From the vast amount of morphological parameters stored for the cell in each image, we chose here to analyze the change in cell area, average optical thickness and cell volume of the mother cell (red) and daughter cells (black and blue, respectively) over time (Figure 3). The cell area for the mother cell decreases markedly, whereas the thickness increases until mitosis. The two daughter cells, on the other hand, show increasing cell area and decreasing thickness. The volume for all cells is relatively uniform (Figure 3).

Pan et al., [28] have exemplified the technique by studying the effects of microgravity-induced bone-loss by using DHI for long-term studies on dividing living cells in culture. A DHM was connected to a superconducting magnet for observing cell division in real-time in living cells under conditions of simulated zero gravity. A large gradient high magnetic field was used to achieve magnetic levitation of murine MLO-Y4 osteocytes inside the bore of a superconducting magnet. A mixed gravity environment inside the bore was achieved which enabled environments with both hypo- as well as hyper-gravity. Quantitative phase contrast images were recorded from cells subjected to zero gravity for a total period of $10 \mathrm{~h}$. Pixel intensity profiles were obtained from recorded phase images. Significant changes in cell division and cell optical thickness was observed under varying gravity conditions, which the authors believe to be caused by microgravity induced reorganization of cell structures such as cytoskeleton, cytosol and organelles.

\subsection{Cell death}

Cell death can be categorized as apoptosis, necrosis or as stages in between [23-24, 29-30]. Here we show two different types of cell death as detected with DHM (HoloMonitor M3, Phase Holographic Imaging AB, PHIAB, Lund, Sweden). DU145 prostate cancer cells and L929 mouse fibroblasts were seeded on IBIDI-micro slides (IBIDI, Martinsried, Germany). After $24 \mathrm{~h}$ of incubation, the cells were treated with $50 \mu \mathrm{M}$ etoposide. The cells were followed from the beginning of treatment and images were captured every four minutes using the HoloMonitor M3. The cells were kept at $37^{\circ} \mathrm{C}$ with a heating plate (IBIDI).

DU145 cells contracted, became dense and rounded up after approximately $4 \mathrm{~h}$ of treatment (Figure 4). Then the cells became very uneven and approximately $5.5 \mathrm{~h}$ (i.e. $330 \mathrm{mi}$ nutes) after the beginning of etoposide treatment, the cells fragmented. Interestingly, the remnants of the cell body contracted into a smaller cell-like structure that resembled an apoptotic body. The fragmentation of the cell is a classic hallmark of apoptosis [30]. 


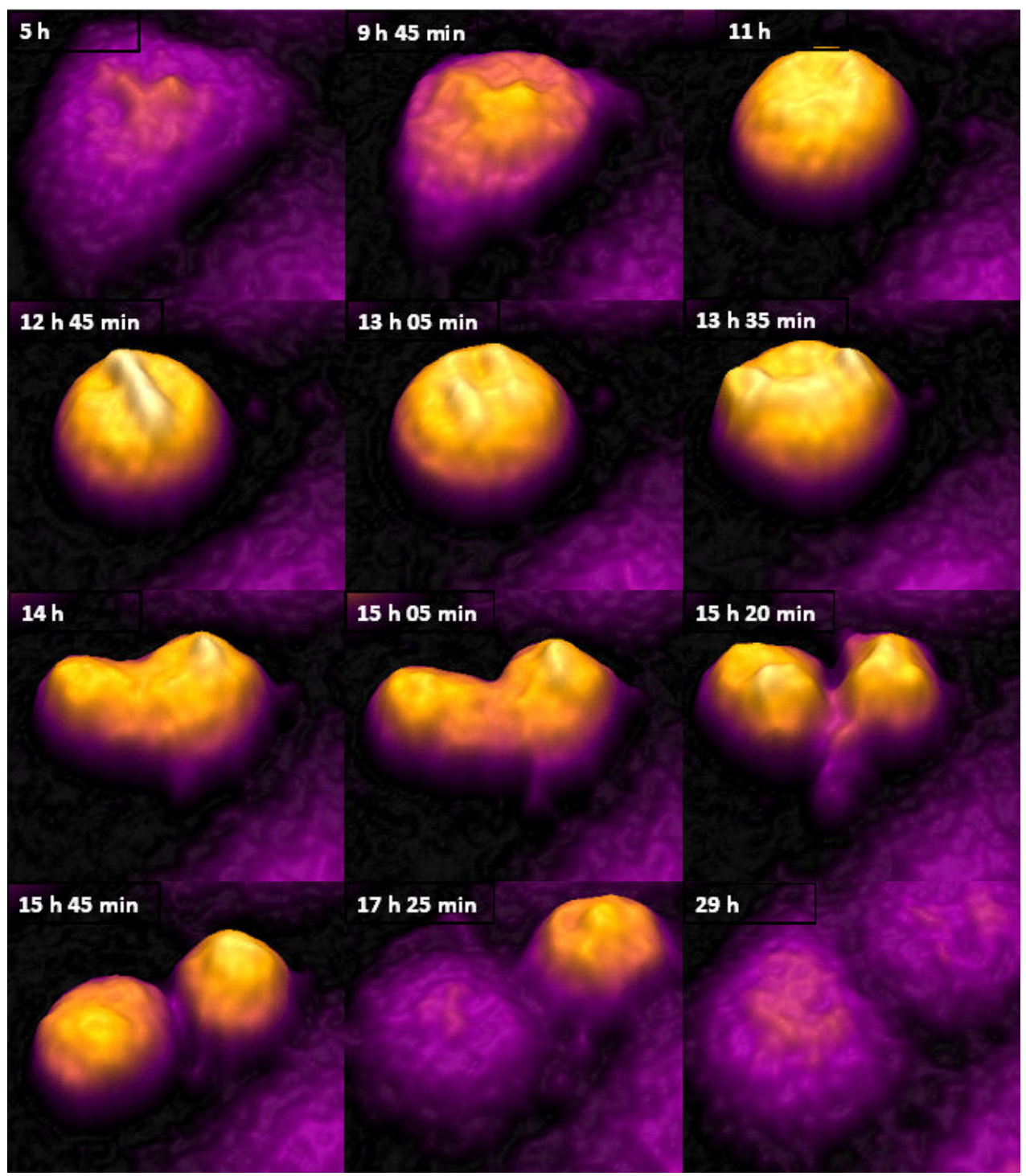

Figure 2. Twelve selected images representing a dividing PanC-1 pancreatic cancer cell from a DHM time-lapse. The image sequence represents $24 \mathrm{~h}$. The images were artificially colored. Note that the interval between the selected images varies, showing the different morphological features of the mitotic events that occur at various rates. As seen in upper part, the mother cell rounds up, the chromatin separates followed by the total division and finally, in the lower part, the two daughter cells achieve the morphology of a mature adherent cell. 

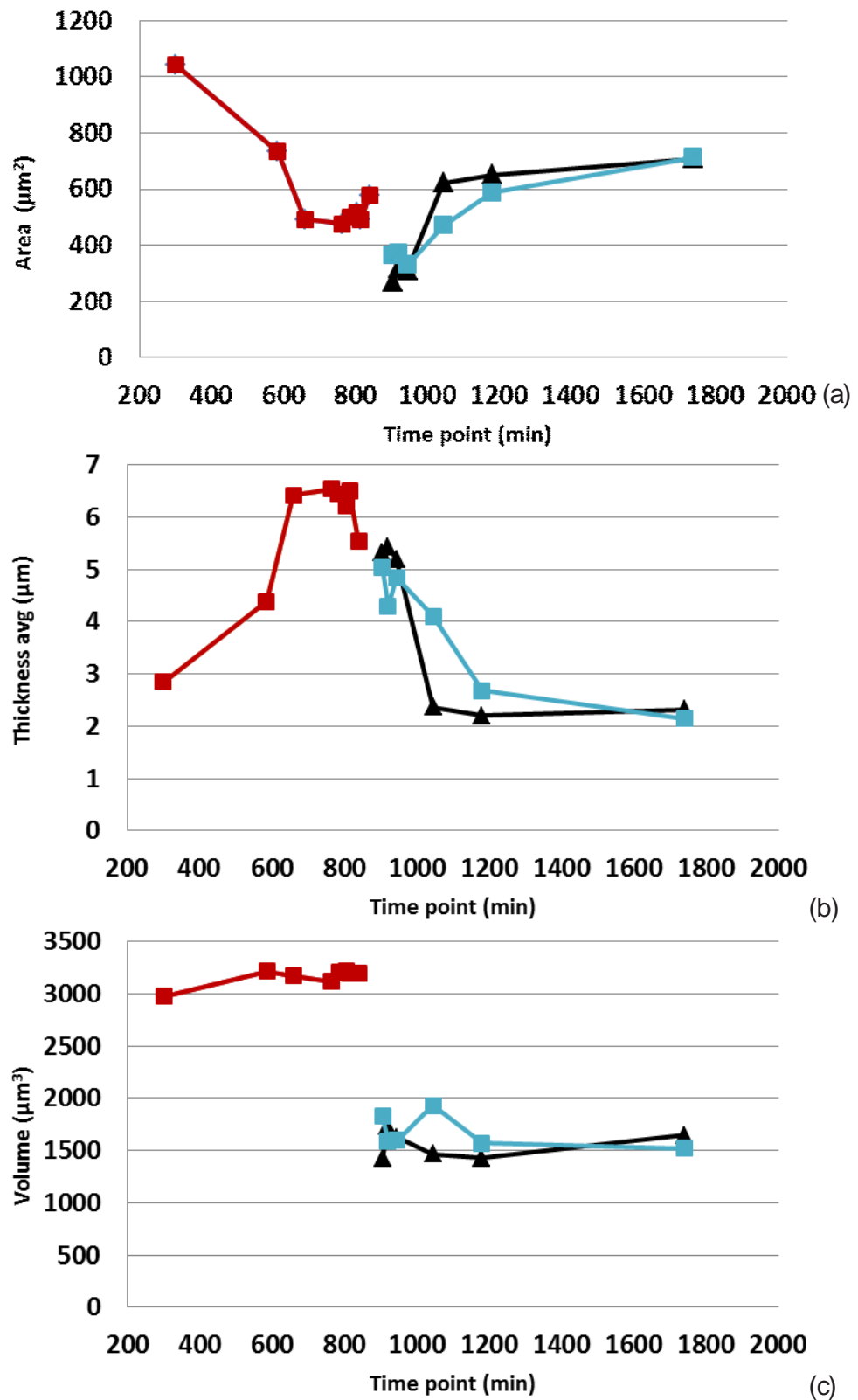

Figure 3. Cell area, thickness and volume define the division of a PanC-1 pancreatic cancer cell into two daughter cells. The mother cell (red) divides after 905 minutes (X-axis, 15 h $05 \mathrm{~min}$ ) and the values for the daughter cells (left cell: black, right cell: blue) are shown at the subsequent time points. 


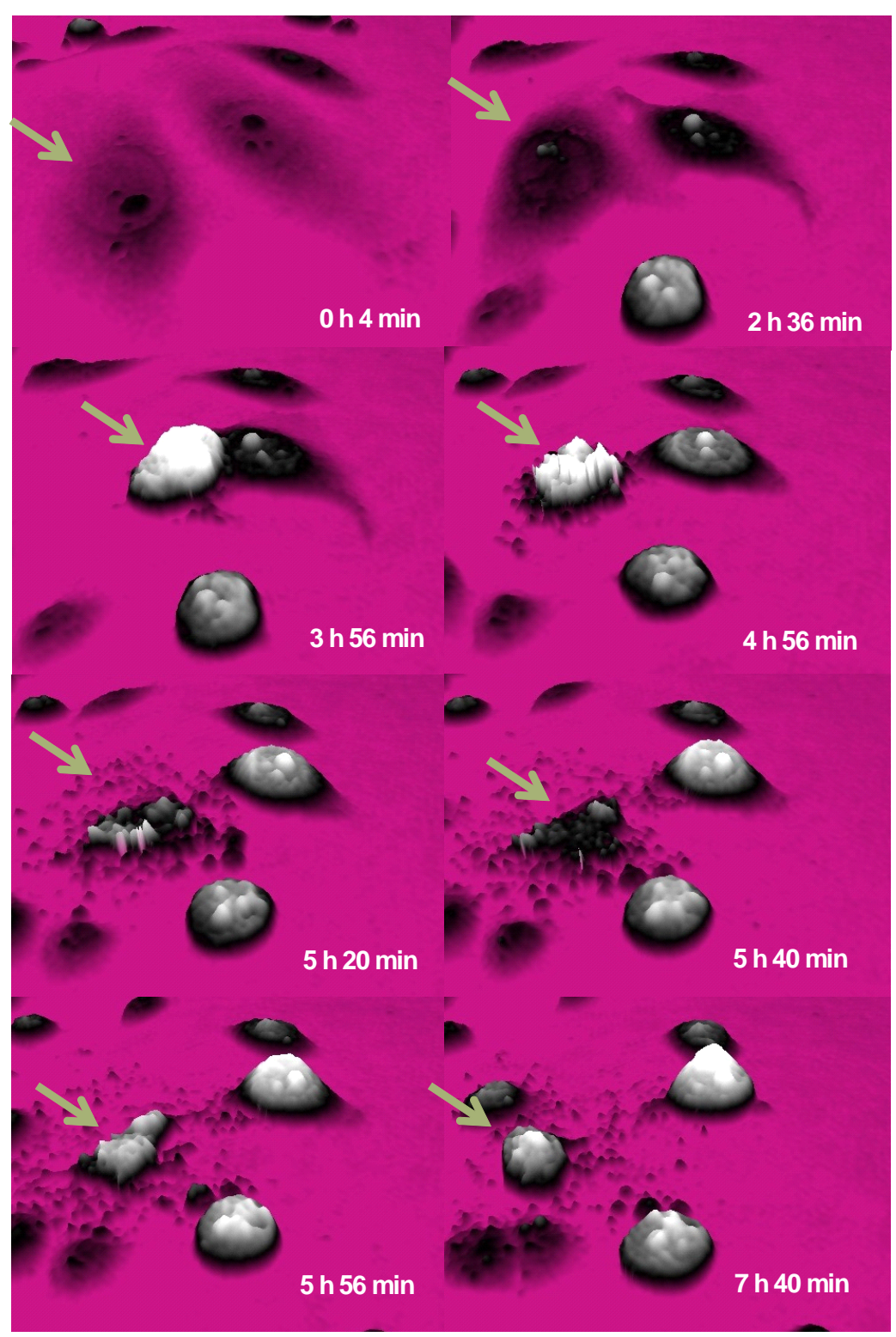

Figure 4. A dying DU145 prostate cancer cell. DHM images were captured every four minutes from the beginning of etoposide treatment $(50 \mu \mathrm{M})$. The green arrows point to a cell, which starts the death process by contracting markedly. The cell then becomes uneven, and breaks apart. The cell death process displays the hallmarks of apoptosis and the cell remnants are gathered in a structure similar to an apoptotic body. 

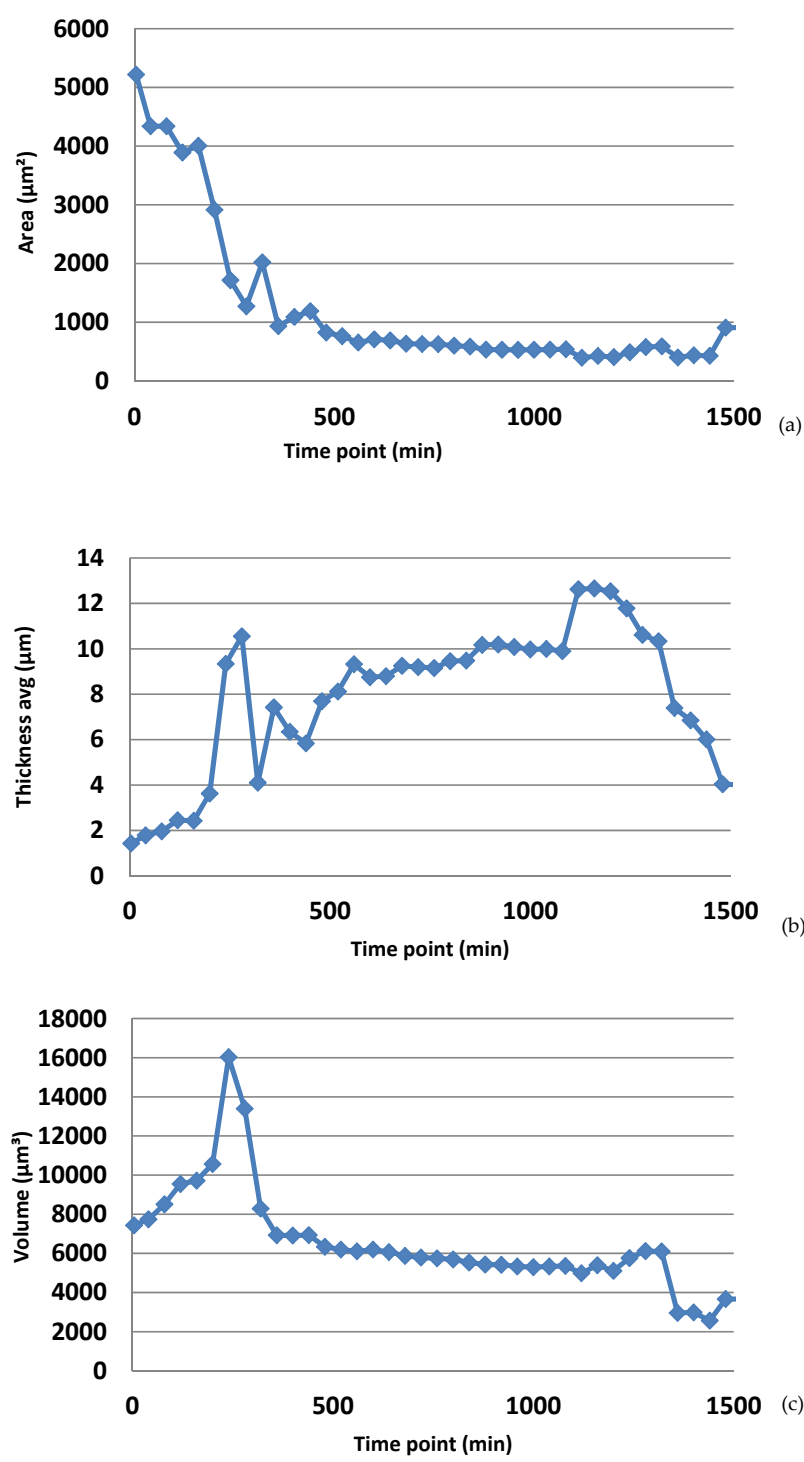

Figure 5. Cell area, thickness and volume changes during etoposide-induced cell death in DU145 cells. The diagrams show how the area, thickness and volume of the DU145 cell in Figure 4 vary over time. The X-axis shows the time after treatment in minutes and the $\mathrm{Y}$-axis shows the measuring units.

When using DHM, each cell can be analyzed regarding morphology. The morphological analysis of the DU145 cell death shown in Figure 4 is displayed in Figure 5. The area of the cell steadily decreased as the cell contracted and rounded up. The cell reached a maximum 
thickness after approximately $4 \mathrm{~h}$ (240 $\mathrm{min})$. The cell volume peaked after approximately 3.5 $\mathrm{h}$, i.e. somewhat later than the thickness, and before the cell fragmented.

The L929 cells behaved very differently. Some cells in this culture started dying within hours of treatment, and others started to die several hours later (Figure 6). The individual cell we followed in this study, started to contract approximately $14 \mathrm{~h}$ after treatment (i.e. 840 minutes). Approximately 50 minutes later, the cell content had become much less optically dense and the cell displayed a morphology that we have previously observed in dead cells (unpublished). After further incubation, the cell remnants became thinner and thinner, and eventually disappeared, as seen in the image where the surrounding cells turn into small cell remnants. The death process was similar for all cells in this sample. It has previously been shown that L929 cell death can take very different forms depending on treatment [31].

The morphological analysis of the L929 cell death shown in Figure 6 is displayed in Figure 7. After $14 \mathrm{~h}$ and 10 minutes (850 minutes) as the cell started to contract, the area decreased and the thickness increased. Thereafter the thickness and volume decreased while the area increased. The dead cell was very thin and large before it started to break down into fragments. The nucleus first condensed and then slowly disintegrated. This particular cell death did not show any of the hallmarks of apoptosis, but rather those of necrosis. We have not confirmed our results with other methods.

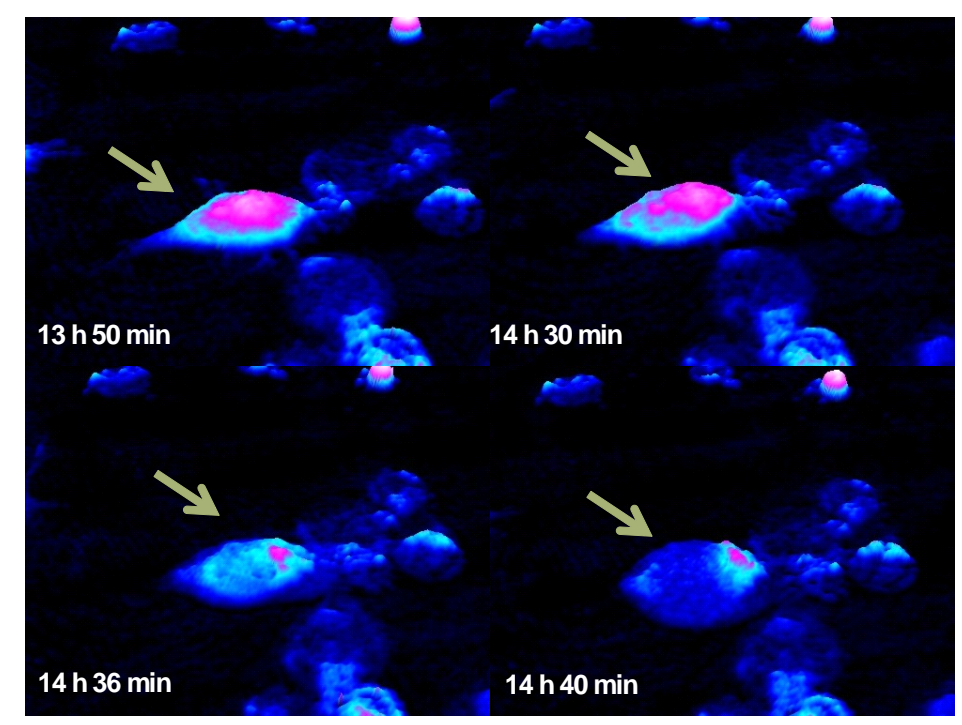

Figure 6. A dying L929 mouse fibroblast cell. Images have been captured every four minutes from the beginning of etoposide $(50 \mu \mathrm{M})$ treatment. The green arrows point to a cell, which starts the death process by contracting slightly. Optically, the cell content becomes thin and finally the cell remnants start to dissolve. The cell death process displays the hallmarks of necrosis. 
The possibility to compare morphological data from individual cells makes it feasible to determine the proportion of dead cells in a culture by capturing an image. If the cells are followed with a timelapse, it is possible to determine the course of cell death, but also to determine the type of cell death.
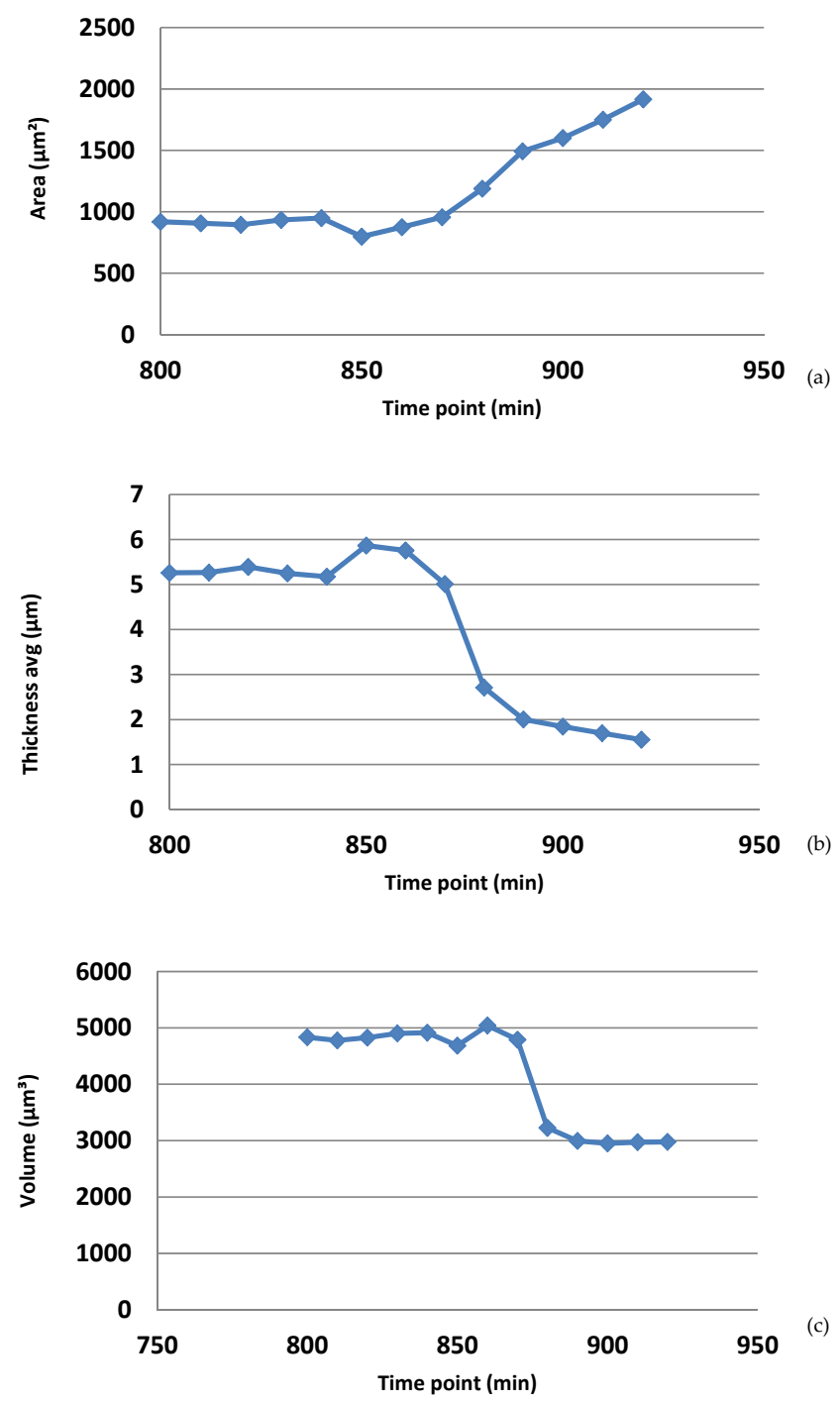

Figure 7. The diagrams show how the area, thickness and volume of the L929 cell in Figure 6 vary over time. The Xaxis shows the time after treatment in minutes and the $Y$-axis shows the measuring units. 


\section{Conclusion}

We present a method to study the morphology of living, dividing and dying cells using DHM. DHM is a non-invasive, non-destructive and non-phototoxic method which allows the user to perform both qualitative and quantitative measurements of living cells over time.

We show here our results on cell division and cell death in single cells. The morphological analyses performed here show changes caused by cell death and cell division, and indicate the possibilities to discriminate between different types of cell death. Cells dying in an apoptosis-like manner display different cell area and cell thickness profiles over time compared to cells dying in a necrosis-like manner, although their volume profiles are very similar. Dividing cells show a characteristic dip in the volume profile, which makes them easily distinguishable. Also, several previous studies show the versatile abilities of DHM. Different cell types have been studied and the morphology has been used to determine cell functionality as well as changes in morphology related to the environment. Cell morphology parameters can be very useful when following the effects of different treatments, the process of differentiation as well as cell growth and cell death. Cell morphology studied by DHM can be useful in toxicology, stem cell and cancer research.

\section{Further research}

Several research groups are now using DHM for cell biological studies. A future goal might be to use the morphological analysis ability of DHM as a fast, automatic, and cost efficient evaluation tool for different cancer treatments. This could make it possible to classify cells and to determine cell morphology and differentiation, cell proliferation, cellular changes of cells transfected with DNA or siRNA, cell death and effects on cell movement, all in a high throughput manner. The traditional methods to detect proteins by cell labelling are all disruptive to the cells both due to the labelling methods and to the photo toxicity caused by the detection methods such as fluorescence microscopy, flow cytometry and multi-well assays. To increase the scope of digital holography it would be useful to develop cell labels that can be detected in a non-invasive, non-destructive way.

\section{Author details}

Kersti Alm ${ }^{1}$ Zahra El-Schich², Maria Falck Miniotis², Anette Gjörloff Wingren²*, Birgit Janicke $^{1}$ and Stina Oredsson ${ }^{3}$

${ }^{*}$ Address all correspondence to: anette.gjorloff-wingren@mah.se

1 Phase Holographic Imaging, Lund, Sweden

2 Biomedical Science, Malmö University, Malmö, Sweden

3 Department of Biology, Lund University, Lund, Sweden 


\section{References}

[1] Carl D, Kemper B, Wernicke G, von Bally G. Parameter-optimized Digital Holographic Microscope for High-Resolution Living-Cell Analysis. Applied Optics 2004;43(36) 6536-6544.

[2] Marquet P, Rappaz B, Magistretti PJ. Digital Holographic Microscopy: a Noninvasive Contrast Imaging Technique Allowing Quantitative Visualization of Living Cells With Subwavelength Axial Accuracy. Optics Letters 2005;30(5) 468-470.

[3] Kemper B, Carl D, Schnekenburger J, Bredebusch I, Schäfer M, Domschke W, von Bally G. Investigations on Living Pancreas Tumor Cells by Digital Holographic Microscopy. Journal of Biomedical Optics 2006;11(3) 34005.

[4] Moon I, Javidi B. Three-Dimensional Identification of Stem Cells by Computational Holographic Imaging. Journal of the Royal Society Interface. 2007; 4(13) 305-313.

[5] Rappaz B, Barbul A, Emery Y, Korenstein R, Depeursinge C, Magistretti P.J, Marquet, P. Comparative Study of Human Erythrocytes by Digital Holographic Microscopy, Confocal Microscopy and Impedance Volume Analyzer. Cytometry Part A 2008;73A(10) 895-903.

[6] Mölder A, Sebesta M, Gustafsson M, Gisselsson L, Gjörloff Wingren A, Alm K. NonInvasive, Label Free Cell Counting and Quantitative Analysis of Adherent Cells Using Digital Holography. Journal of Microscopy 2008;232(2) 240-247.

[7] Kemper B, Bauwens A, Vollmer A, Ketelhut S, Langehanenberg P, Müthing J, Karch H, von Bally G. Label-Free Quantitative Cell Division Monitoring of Endothelial Cells by Digital Holographic Microscopy. Journal of Biomedical Optics 2010;15(3): 036009 .

[8] Persson J., Mölder A., Pettersson S.G., Alm K. Cell Motility Studies Using Digital Holographic Microscopy In: A. Méndez-Vilas and J. Díaz Álvarez (Eds.) Microscopy: Science, Technology, Applications and Education. Formatex Research Center. 2010. p1063-1072.

[9] Alm K, Cirenajwis H, Gisselsson L, Gjörloff Wingren A, Janicke B, Mölder A, Oredsson S, Persson J. Digital Holography and Cell Studies. In: Joseph Rosen (ed) Holography, Research and Technologies, In Tech, 2011. p237-252.

[10] El-Schish Z., Mölder A., Sebesta M., Gisselsson L., Alm K., Gjörloff Wingren, A. Digital holographic microscopy - innovative and non-destructive analysis of living cells. In: A. Méndez-Vilas and J. Díaz Álvarez (Eds.) Microscopy, Science, Technology, Applications and Education. Formatex Research Center. 2011. p1055-1062.

[11] Memmolo P, Di Caprio G, Distante C, Paturzo M, Puglisi R, Balduzzi D, Galli A, Coppola G, Ferraro P. Identification of Bovine Sperm Head for Morphometry Analysis in Quantitative Phase-Contrast Holographic Microscopy. Optics Express 2011;19(23) 23215-23226. 
[12] Crha I, Zakova J, Huser M, Ventruba P, Lousova E, Pohanka M. Digital Holographic Microscopy in Human Sperm Imaging. Journal of Assisted Reproduction and Genetics. 2011;28(8) 725-729.

[13] Mihailescu M, Scarlat M, Gheorghiu A, Costescu J, Kusko M, Paun IA, Scarlat E. Automated Imaging, Identification, and Counting of Similar Cells From Digital Hologram Reconstructions. Applied Optics. 2011;50(20) 3589-3597.

[14] Jin W, Wanga Y, Ren N, Bu M, Shang X, Xu Y, Chen Y. Simulation of Simultaneous Measurement for Red Blood Cell Thickness and Refractive Index. Optics and Lasers in Engineering. 2011;50 154-158.

[15] Kemper B, Barroso A, Woerdemann M, Dewenter L, Vollmer A, Schubert R, Mellmann A, von Bally G, Denz C. Towards 3D Modelling and Imaging of Infection Scenarios at the Single Cell Level Using Holographic Optical Tweezers and Digital Holographic Microscopy. Journal of Biophotonics. 2012; doi:10.1002/jbio.201200057.

[16] Pavillon N, Kühn J, Moratal C, Jourdain P, Depeursinge C, Magistretti PJ, Marquet P. Early Cell Death Detection With Digital Holographic Microscopy. PLoS One. 2012;7(1):e30912. doi:10.1371/journal.pone.0030912

[17] Jourdain P, Pavillon N, Moratal C, Boss D, Rappaz B, Depeursinge C, Marquet P, Magistretti PJ. Determination of Transmembrane Water Fluxes in Neurons Elicited by Glutamate Ionotropic Receptors and by the Cotransporters KCC2 and NKCC1: A Digital In-Line Holographic Microscopy Study. Journal of Neuroscience. 2011:31(33) 11846-11854.

[18] Jericho MH, Kreuzer HJ, Kanka M, Riesenberg R. Quantitative Phase and Refractive Index Measurements With Point-Source Digital In-Line Holographic Microscopy. Applied Optics. 2012;51(10) 1503-1515.

[19] Rappaz B, Marquet P, Cuche E, Emery, Y, Depeursinge C, Magistretti PJ. Measurement of the Integral Refractive Index and Dynamic Cell Morphometry of Living Cells With Digital Holographic Microscopy. Optics Express. 2005;13(23) 9361-9373.

[20] Barer R. Refractometry and Interferometry of Living Cells. Journal of the Optical Society of America. 1957;47(6) 545-556.

[21] Bishara W, Sikora U, Mudanyali O, Su TW, Yaglidere O, Luckhart S, Ozcan A. Holographic Pixel Super-Resolution in Portable Lensless On-Chip Microscopy Using a Fiber-Optic Array. Lab on a Chip. 2011;11(7) 1276-1279.

[22] Chalut KJ, Ekpenyong AE, Clegg WL, Melhuish IC, Guck J. Quantifying Cellular Differentiation by Physical Phenotype Using Digital Holographic Microscopy. Integrative Biology:Quantitative Biosciences From Nano To Macro. 2012;4(3):280-284.

[23] Reed JC. Mechanisms of Apoptosis. American Journal of Pathology. 2000;157(5) 1415-1430.

[24] Kroemer G, Galluzzi L, Vandenabeele P, Abrams J, Alnemri ES, Baehrecke EH, Blagosklonny MV, El-Deiry WS, Golstein P, Green DR, Hengartner M, Knight RA, Ku- 
mar S, Lipton SA, Malorni W, Nunez G, Peter ME, Tschopp J, Yuan J, Piacentini M, Zhivotovsky B, Melino G. Classification of Cell Death: Recommendations of the Nomenclature Committee on Cell Death. Cell Death and Differentiation. 2009;16(1) 3-11.

[25] Martin SJ, Reutelingsperger CP, McGahon AJ, Rader JA, van Schie RC, LaFace DM, Green DR. Early Redistribution of Plasma Membrane Phosphatidylserine is a General Feature of Apoptosis Regardless of the Initiating Stimulus: Inhibition by Overexpression of Bcl-2 and Abl. Journal of Experimental Medicine. 1995;182(5) 1545-1556.

[26] Khmaladze A, Matz RL, Epstein T, Jasensky J, Banaszak Holl MM, Chen Z. Cell Volume Changes During Apoptosis monitored in Real Time Using Digital Holographic Microscopy. Journal of Structural Biology. 2012;178(3):270-278.

[27] Logg K, Bodvard K, Blomberg A, Käll, M. Investigations on Light-Induced Stress in Fluorescence Microscopy Using Nuclear Localization of the Transcriptionfactor Msn2p as a Reporter. Yeast Research. 2009;9(6) 875-884.

[28] Pan F, Liu S, Wang Z, Shang P, Xiao W. Digital Holographic Microscopy Long-Term and Real-Time Monitoring of Cell Division and Changes Under Simulated Zero Gravity. Optics Express. 2012;20(10) 11496-11505.

[29] Formigli L, Papucci L, Tani A, Schiavone N, Tempestini A, Orlandini GE, Capaccioli S, Orlandini SZ. Aponecrosis: Morphological and Biochemical Exploration of a Syncretic Process of Cell Death Sharing Apoptosis and Necrosis. Journal of Cellular Physiology. 2000;182(1) 41-49.

[30] Elmore S. Apoptosis: A Review of Programmed Cell Death. Toxicologic Pathology. 2007;35(4) 495-516.

[31] Humphreys DT, Wilson MR. Modes of L929 Cell Death Induced By TNF-Alpha and Other Cytotoxic Agents. Cytokine. 1999;11(10) 773-782. 
